\title{
Application of Norms Models with Vectoral System in Construction Projects
}

\author{
Vladimir Križaić \\ Construction VET Secondary School, Community College of Međimurje County, Čakovec HR-40000, Croatia
}

\begin{abstract}
Normization, i.e., the system of norms is a structure that defines the group of elements containing the norm values for the requirements of a certain resource. Resources comprise of materials, machines and labor. All the requirements of the measure units of the resources are given statically and with the discrete data. Thus, every slight change in the expense list item reference causes a change in norm and our norm is not flexible and features a discrepancy with the real life situations. In order to achieve a higher level of preciseness and to speed up the technological processes of planning and norming the engines of a company that lead to the regulation of the system, the discrete elements of the working (time-related) norms should be replaced by the dynamic ones. This is made possible through setting up norms models that in turn can be presented by formulae in the vectoral system. The use and implementation of the new technologies in terms of production, computer science and cybernetics provides for upgrading the norm requirements. New working tasks in turn require a new norm standardization, which can be applied to the hydrodemolition of concrete constructions by means of water robots that use high pressure water jets.
\end{abstract}

Key words: Norms models, water robots, model standardization norm, Erlang distribution.

\section{Introduction}

By means of the model standardization and the dynamic defining of the organizational needs [1, 2], the static project of organizing construction works is turned into a dynamic one. Dynamics is in turn defined by mathematical and statistical methods applied to particular models of various construction operations. In order for the company to increase profit, i.e., for the water robot economic indices to increase, the technical efficiency of a particular robot was observed in the field, namely in the course of actual works performed at refurbishment of a bridge on a section of the Zagreb detour motorway section.

The function inverse to efficiency is the norm function. For the defining of any work, the respective discrete norm is defined with the accompanying working, material and machinery resources.

Thus, the infinity and the lack of quality of being

Corresponding author: Vladimir Križaić, M.Sc., lecturer, chartered engineer, research fields: organization and technology. E-mail: Vladimir.Krizaic@gmail.com. defined cannot be seen for all the differences in the variables that the norm relates to in practice and especially not for the variables concerning working (time) resources.

Depending on the dimension given by the documentation and the particular situation in the field, the averaged constant of the discrete norm does not match the requirements implied by a more productive way of performing operations and this is especially so for new technologies that are ahead of the norm technologies. The most considerable mismatches in that sense are featured by the time working resource that is usually just averaged approximate and mostly obsolete efficiency. If the technology of demolition [3] by means of robots producing a high-pressure water jet is closely observed and if the respective literature is consulted, the efficiency of the robots (developed from a Swedish innovation-Fig. 1) turns to amount an average of $0.35-1.5 \mathrm{~m}^{3} / \mathrm{h}$, depending on the type of the robot and the hardness of concrete.

As the machine is operated by two engineers, the overall norm amounts from $1.3 \mathrm{~m}^{3} / \mathrm{h}$ to $6 \mathrm{~m}^{3} / \mathrm{h}$. The 


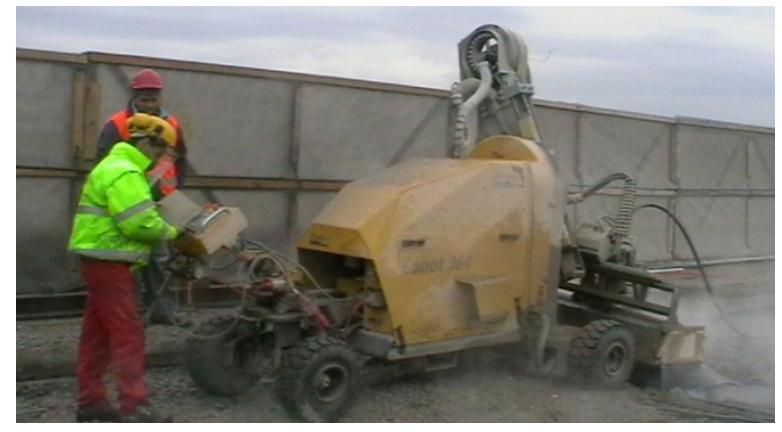

Fig. 1 Conjet robot (at operation).

discrete norm of $3 \mathrm{~m}^{3} / \mathrm{h}$ is usually used for the theoretical calculations [4].

The hydrodemolition technology is rarely mentioned in our magazines [5].

\section{Method-Vector Norm}

The analysis and systematization of the old norms bring to light the variables of construction, technology and organization needed for defining the vector norm hypothesis (Eq. (1)).

$$
\begin{aligned}
\text { Vector norm } & =N_{k, r, k o_{k}, k d_{i}} \\
& =k d_{i}\left(k o_{k}\left(f_{0}(k, r)\right)\right)
\end{aligned}
$$

There are tables for the envelope technology example showing the functions of resources $(r)$ (i.e., the technology of performance), the construction being processed $(k)$ and the dimensions of the construction $\left(k d_{i}\right)$ influencing not only the time resource but the material component of the norm as well. By means of the use of these variables and defining the basic construction type $\left(k o_{k}\right)$, all the discrete tabular presentations of the envelope systems can be boiled down to a graphic vector image (Fig. 2). If the construction as mentioned above is excluded, on the grounds that it is the easiest to define and most frequently defined, the basic construction is defined and furtherly used for defining other constructions via respective coefficients. So as to define the equation for the time span for the basic construction from numerous discrete values at given resources, the Gaussian minimum mean square method is the most appropriate one in Eqs. (2) and (3). It gives the approximation of the parameters, i.e., the model standardization norm for the basic envelope system in Eq. (4) for either linear or non-linear equation systems (Fig. 2) [6, 7].

$$
\begin{aligned}
& f(r)=a+b r+c r^{2} \equiv M S N \\
& K=T \times D^{-1}
\end{aligned}
$$

where, $K$-coefficients, $T$-norm, $D$-dimensions (resource level), $a, b, c$-dimensionless coefficient:

$$
\begin{aligned}
& {\left[\begin{array}{l}
a \\
b \\
c
\end{array}\right]=\left[\begin{array}{c}
\sum_{i} t_{i} \\
\sum_{i} t_{i} \times r \\
\sum_{i} t_{i} \times r_{i}^{2}
\end{array}\right] \times\left[\begin{array}{ccc}
n_{i} & \sum_{i} r_{i} & \sum_{i} r_{i}^{2} \\
\sum_{i} r_{i} & \sum_{i} r_{i}^{2} & \sum_{i} r_{i}^{3} \\
\sum_{i} r_{i}^{2} & \sum_{i} r_{i}^{3} & \sum_{i} r_{i}^{4}
\end{array}\right]^{4}} \\
& T=f(r)=1.05+0.12 r-0.08 r^{2}
\end{aligned}
$$

Materials, equipment and transport are in function of the constructive and geometric features of a certain construction endeavor and the above mentioned technology $[8,9]$. Thus, the materials being spent, by means of relating the constructional equations to the resource efficiency, are boiled down to the dimensions of the drawings. The results of the relating are the monograms of the bearing capacities of constructions and serve at the same time as the norm for resources used for a certain construction [10]. The tendency of making procedures shorter and performing the resource overview quicker resulted in the vector description of norms, i.e., the VMSN (vector model standardization norm) in Eq. (5) for the purpose of constructing the wall envelope system in Eq. (6) (Fig. 3):

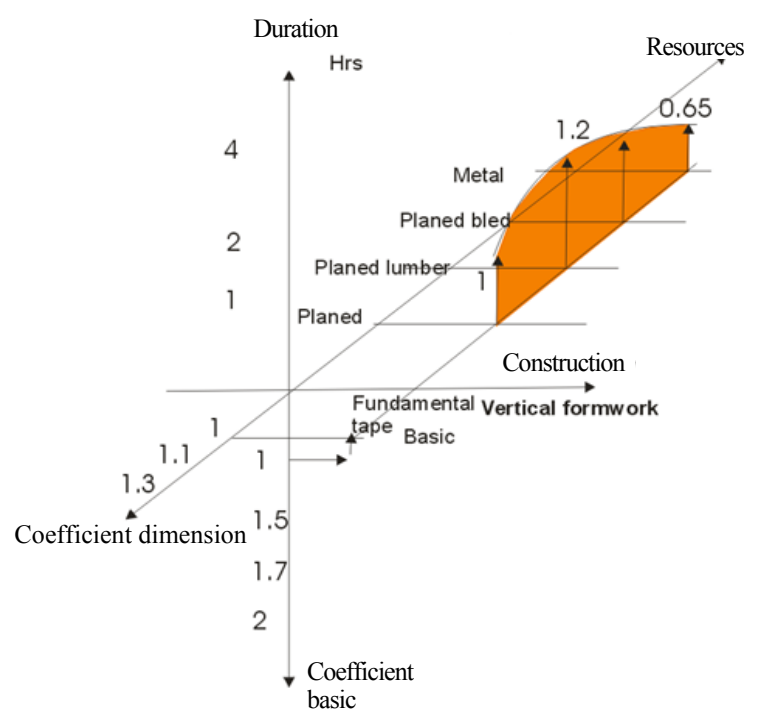

Fig. 2 Functional norms dependency on resources. 


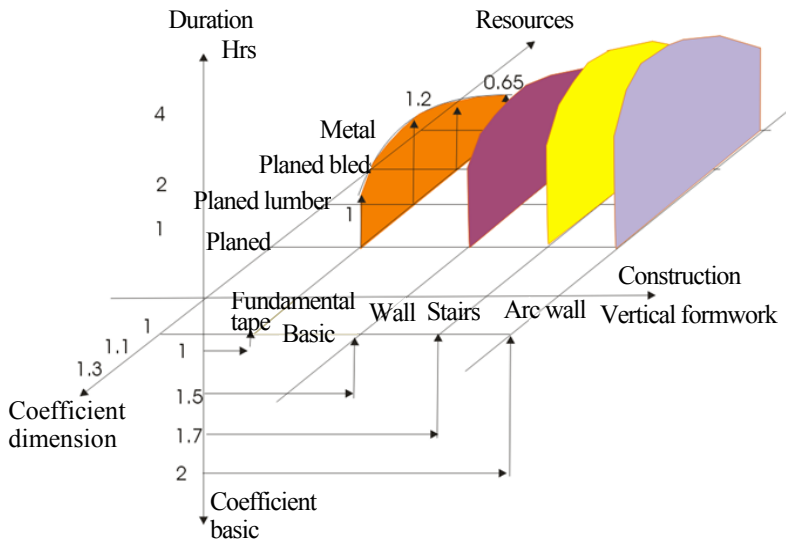

Fig. 3 VMNS (vectoral norms) for the envelopes of vertical constructions as performed by various construction companies.

$$
\begin{gathered}
N_{k, r, k o_{k}, k d_{i}}=\sum_{k d_{i}} k d_{i}\left(\sum_{k o_{k}} k o_{k}\left(\sum_{k} \sum_{r} f_{o}(k, r)\right)\right) \\
N_{k, r, k o_{k}, k d_{i}}=k d_{i} 1.5\left(1.05+0.12 r-0.08 r^{2}\right)
\end{gathered}
$$

Thus, the equation from the hypothesis (1) proved correct.

\section{Results-Vector Norm of Robotic Hydrodemolition}

The classical approach to the defining of the practical efficiency of a machine via defining the practical efficiency of a robot could be said to be inappropriate and complicated as the defining of all forces and resistances will imply performing of some additional research. Therefore, based on the monitoring of a typical construction site, namely the restoration of a bridge over the Sava River on the Zagreb bypass section of the motorway at Ivanja Reka, the data were used for a statistics analysis of the standards of operating for Conject robots. The various monitored variables of operating for Conject robots 361, 362 and 364 are shown in Table $1[11,12]$.

When the given database is sorted in accordance with the given variables, it turns out that the efficiency depends on the depth of hydrodemolition that in turn depends on the sort of concrete. Working in shifts does not play part in the efficiency and the width of the processed area is as well considered as the depth. The density of the efficiency frequency is plausibly shown by means of the Erlang distribution that stemmed from
Table 1 Data obtained from on-site monitoring of Conject

\begin{tabular}{|c|c|c|c|c|c|c|}
\hline Pump & Robot & $\begin{array}{l}\text { Machinist } \\
\text { (2) }\end{array}$ & Operation & $\begin{array}{l}\text { Volume } \\
\left(\mathrm{m}^{3}\right)\end{array}$ & $\begin{array}{l}\text { Hour } \\
(\mathrm{h})\end{array}$ & Effect \\
\hline 333 & 361 & Kljajić & poz 5/7 & 6.7 & 11 & 0.61 \\
\hline 364 & 364 & Damjan & poz 5/7 & 2.9 & 3 & 0.96 \\
\hline 403 & 302 & Miličević & poz 5/7 & 5 & 5 & 1 \\
\hline 333 & 361 & Ozimec & poz 5/7 & 6.3 & 12 & 0.53 \\
\hline 364 & 364 & Palijan & poz 5/7 & 6.6 & 11 & 0.6 \\
\hline 403 & 302 & Kovačić & poz 5/7 & 5.3 & 9 & 0.59 \\
\hline 353 & 361 & Kljajić & poz 5/7 & 1.2 & 4 & 0.29 \\
\hline
\end{tabular}
robots operation.

the binomial limited distribution, i.e., from the Poisson distribution. The mathematical expectation or arithmetic mean, or technically speaking, the weight point of the Erlang distribution will suffice to determine the average robot efficiency. The mathematical expectation of the continuous Erlang curve is larger than the discrete distribution as the constant line fills the blank spaces of the discrete distribution in Eq. (7) [13].

$$
E\left(y_{x}\right)=\int_{-\infty}^{\infty} x\left(y_{x}\right) d x>\sum_{i} \frac{x_{i} y_{i}}{\sum_{i} y_{i}}
$$

The expectation of the Erlang distribution is already known, i.e., Eq. (8):

$$
E\left(y_{x}\right)=\frac{k+1}{\lambda} ; y_{x}=\lambda \frac{(\lambda x)^{k}}{k !} e^{-\lambda x}
$$

where, the $k$ and $\lambda$ parameters are the discrete values of a sample for whose values the Erlang function is approximately the same as the distribution obtained from the monitoring of a certain robot.

By means of the use of the MathCad software and by means of assigning certain values to the parameters, the curve is observed and assessed in terms of its fitting/not fitting into our selective distribution pattern. When the graph approximately fits, the parameters are taken as finite ones and they in turn instantly define the mathematical efficiency expectation for (i.e., the discrete average efficiency of) a robot. The influence of the parameters on the curve flow is as follows: the $k$ parameter shifts the curve to the right side with a peak increase, whereas the increase or decrease of the $\lambda$ parameter makes the curve narrower or wider, respectively. For the purpose of monitoring the 
frequency, the frequency coefficient $k f$ is deployed and its increase makes the curve peak higher in Eq. (9).

$$
y_{x}=\lambda \frac{(\lambda x)^{k}}{k !} e^{-\lambda x} k f
$$

The $k f$ coefficient does not influence the expectancy but it influences the ordinate as shown in Fig. 4.

A graphic presentation of the Erlang distributions for the above is mentioned bridge over a month time span (Table 2, Figs. 4 and 5).

If the Erlang distributions are tested by $\chi^{2}$ for $\alpha=$ 0.05 and 2 freedom degrees, the curves tested are valid for a random sample as the discrepancy between the empiric distribution and the Erlang distribution are not significant.

A tabular presentation of the efficiency expectancies for the robots is monitored (Table 2).

The substitutes for graphs themselves are the equations of the basic and other graphs, i.e., of hydrodemolition of decks, beams or vertical constructions. Thus, the basic deck hydrodemolition graph is Eq. (10):

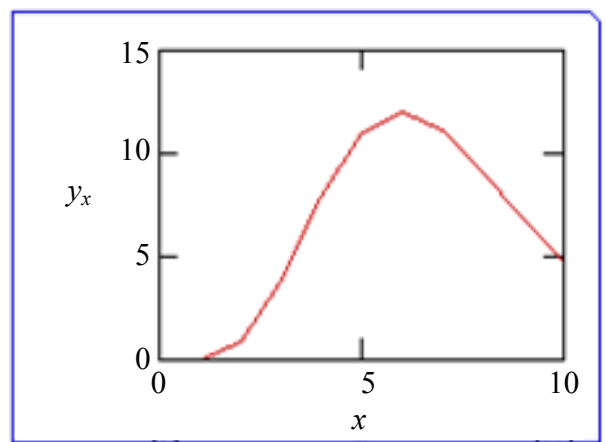

(a)

$$
U o_{k, r, k o_{k}, k d}=k d_{c} k o_{k} U o_{k, r}
$$

where, $k d$ is the coefficient of the construction $c$ dimension for a type of concrete and of the basic coefficient that is the function of the construction and the efficiency equation that is in the function of resources and the construction. As to the basic efficiency, the coefficients are of measure unit quality and thus, the equation gets the form of the function of the basic efficiency in Eq. (11).

$$
U o_{k, r, k o_{k}, k d}=U o_{k, r}=-0.1 r+0.8
$$

The construction efficiency equations are identical to the basic efficiency, granted that the coefficients are different whereas the basic efficiency function remains the same. If multiplied one by another, the result gives the efficiency values expressed in cubic meters per hour $\left(\mathrm{m}^{3} / \mathrm{h}\right)$ in Eq. (12).

$$
U_{k, r, k o_{k}, k d}=k d_{c} k o_{k} U o_{k, r}
$$

The arithmetic mean of a series of sequential observations gives the average resource efficiency that in turn becomes a variable of the vector efficiency.

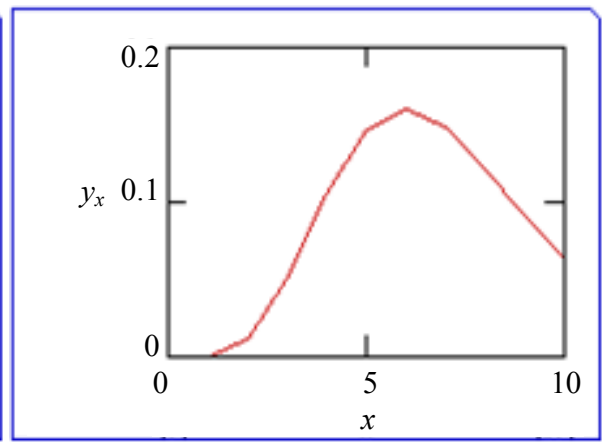

(b)

Fig. 4 The influence of $k f$ on the Erlang distribution (a) with and (b) without $k f$.

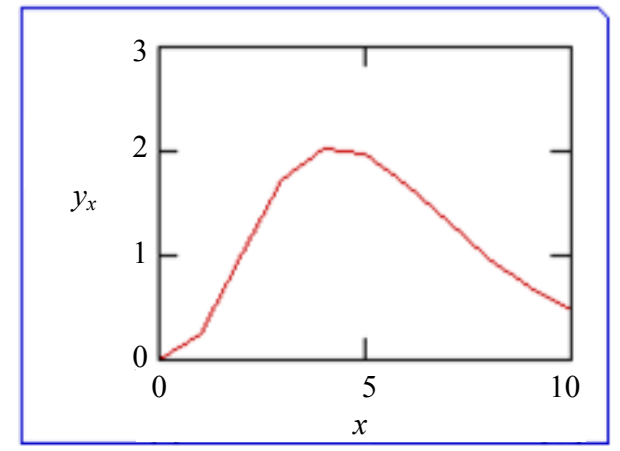

(a)

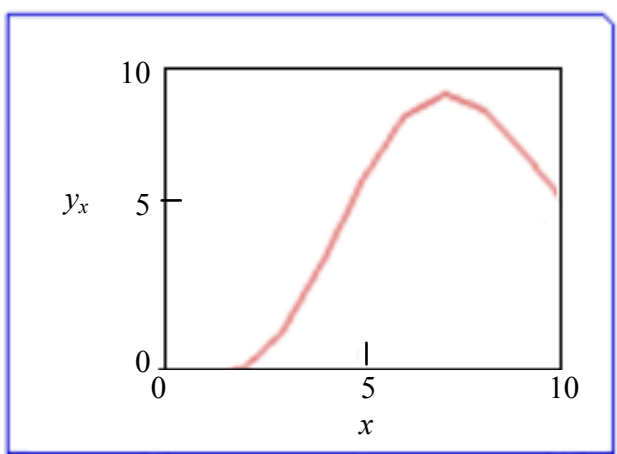

(b)

Fig. 5 The Erlang distribution for a 361 robot for (a) $d>20 \mathrm{~cm}$ and (b) $d \leq 20 \mathrm{~cm}$. 
Table 2 The expectancy (efficiency) for robots in the course of November.

\begin{tabular}{llllll}
\hline \multirow{2}{*}{ Robot } & \multirow{2}{*}{$\mathrm{E}(\mathrm{x})($ Efficiency $(x))$} & \multicolumn{2}{c}{ For robots } & $\bar{X}$ (Arithmetic mean of $(x))$ & $\begin{array}{l}\chi^{2}\left(\text { Hi test } \chi^{2}<\chi_{\alpha}^{2}\right) \\
\chi_{\alpha}{ }^{2}=5.99\end{array}$ \\
\cline { 3 - 5 } & & $d \leq 20 \mathrm{~cm}$ & $d>20 \mathrm{~cm}$ & & 5.75 \\
361 & 0.7 & 0.8 & 0.48 & 0.54 & 2.84 \\
364 & 0.6 & 0.65 & 0.45 & 0.56 & 5.96 \\
\hline
\end{tabular}

As to the observations related to the depth, they are dependant of the concrete type. It can be seen that for $d>20$ the efficiency is of approximately constant value with beams made of the stabile concrete type C 40/50, whereas with thinner decks, where the concrete has deteriorated, there is a dispersion of efficiency at the same rate as the rate of the concrete hardness dispersion. This leads to the definition of the functional dependency of the dimension coefficient (concrete hardness $C$ ) that is presented by the given linear function in Eq. (14) for the zero-abscise for C $40 / 50$ and with respective given coefficients $k_{c}$ (Fig. 6).

$$
\begin{gathered}
N_{k, r, k o_{i}, k d}=n_{r} \cdot 1 / U_{k, r, k o_{k}, k d}=n_{r} / k d_{c} k o_{k} U o_{k, r} \\
k_{c}=0.15 x+0.7
\end{gathered}
$$

The relation between the two occurences of the given concrete hardness in the $5 / 7$ deck in dependency with the 361 robot efficiency gives the curve in Eq. (15):

$$
k_{c}=\left(0.35+0.13 x+0.01 x^{2}\right) n
$$

By means of the correlation theory of the given regression curves in Eqs. (14) and (15), there is the proof of the dependency of the coefficient on the concrete hardness in line with the given curves with the linearity of $n$ and the correlation coefficient of 0.52 that is widely used in practice.

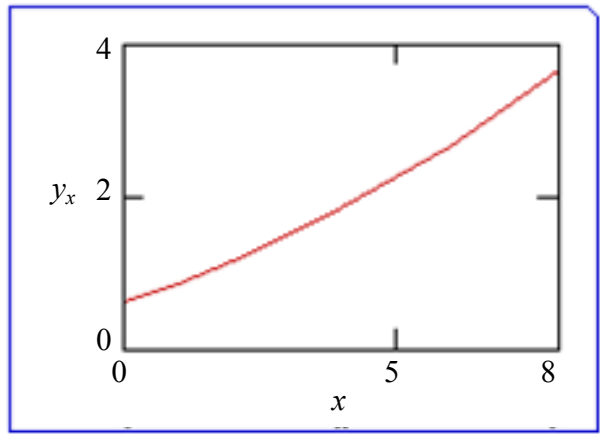

Fig. 7 The values of the coefficient $k_{c}$.
As the proof for the mentioned equation, the real life data on the bridge deck hardness [14] can be used. The percent of the share of $C$ in the deck should correspond to the percentage of the area, i.e., it is to correspond on accordance with the given expectation of the efficiency frequency (Fig. 5).

This in turn provides for establishing the average coefficient of the deck concrete hardness dimension that amounts 0.85 for $\mathrm{C} 30 / 40$ in accordance with the design (Fig. 7), it, however, amounts approximately 1.07 for the real life data as calculated by means of the Eq. (16).

$$
\mathrm{E}\left(k_{c}\right)=\frac{1}{100} \sum_{c} \% F_{c} \cdot k_{c}
$$

The obtained data provides for an optimal hydrodemolition planning process in the construction industry. The procedure as well reversely explains the

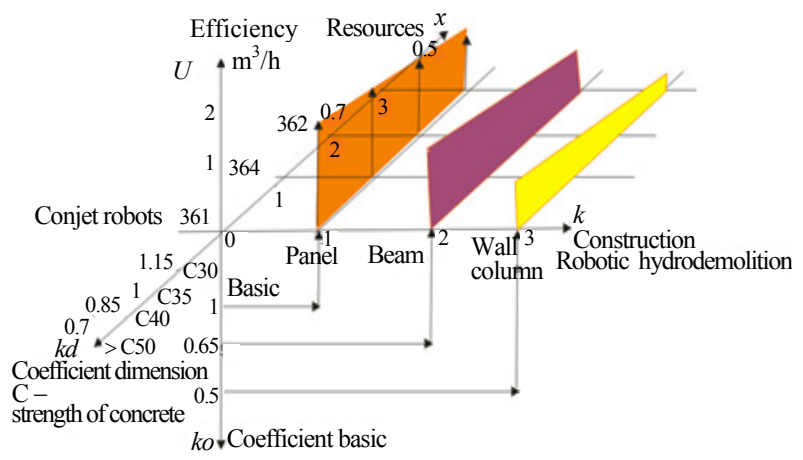

Fig. 6 The vectoral efficiency of hydrodemolition by Conjet robots.

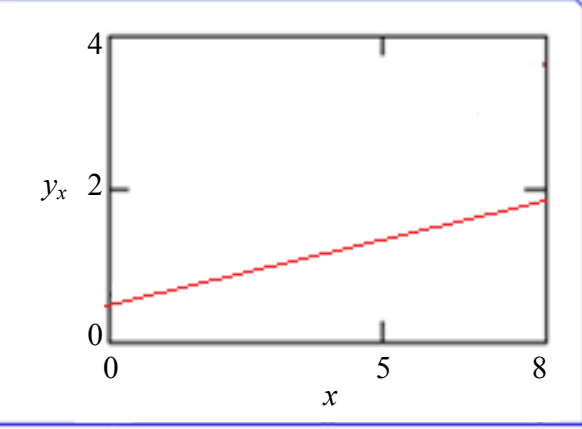




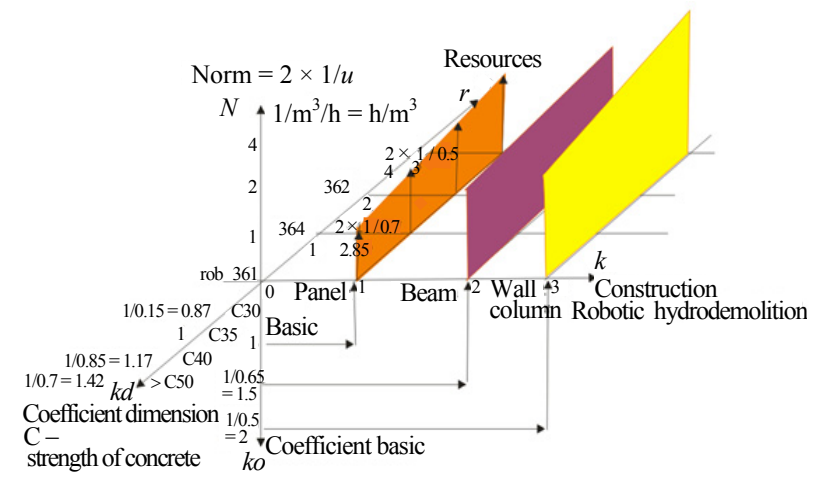

Fig. 8 Vectoral norm of robotic hydrodemolition.

Table 3 The real average efficiency of a robot at Zaprešić.

\begin{tabular}{lll}
\hline Robot & Frequency & $\bar{X}$ \\
\hline 361 & 4 & 0.69 \\
362 & 26 & 0.72 \\
364 & 36 & 0.8 \\
\hline
\end{tabular}

estimated efficiency as dependent of $C$ that amounts 0.75 whereas there has been the value of $0.8 \mathrm{~m}^{3} / \mathrm{h}$ with robot 361 in practice and in accordance with the Erlang distribution.

This method was tested in another research concerning the flyover above the railroad line at Zaprešić (a suburb of the Croatian capital Zagreb). By means of the reverse method of measuring the deck hardness by a sclerometer, a series of interesting data was obtained through which the efficiency of resources (i.e., robots) can be predicted. The data processing resulted in the Erlang distribution ranging from $\mathrm{C} 29$ to C41 with the substitute coefficients $k=6, \lambda=1$ and $k f=$ 75. As for the given parameters, the expected concrete deck hardness is $\mathrm{C} 36$, whereas, for the given hardness, the robot efficiency is estimated at $0.68 \mathrm{~m}^{3} / \mathrm{h}$ via the given vectoral presentation of efficiency(Fig. 8). It is interesting that the processing of all data from the daily reports on the robot hydrodemolition efficiency resulted in Table 3, where the interval ranges of the robot efficiency amounts from $0.6 \mathrm{~m}^{3} / \mathrm{h}$ to $1.08 \mathrm{~m}^{3} / \mathrm{h}$. The efficiency was higher where hydrodemolition was deployed on smaller depths.

\section{Conclusions}

The discovery and modeling of functional dependencies concerning certain type of work provide for a constant process of modernization and standardization of norms. A further input of data into the construction site database and the processing of these data lead to optimal results, although the on-site instant processing is already optimal nowadays. In the same manner in which a function can be modeled for the time component of the working resource, other possible resource requirement can be modeled as a function of designers' equations or engineering or other scientific achievements regarding equations relating to the material or engineering or transport requirements of the resource. While creating a relation with the structural programing, which is a significant support to the given modeling process, the classical static norming is transformed into a dynamic one. By means of this programing (DSP (dynamic structural programing)), an organization dealing with construction can make a giant leap in terms of the technologic development and reduce the respective gaps at the same time [15-17].

\section{References}

[1] V. Križaić, Model standardization, in: 6th International Gathering on Building Economics, Zagreb, 1996.

[2] V. Križaić, Model standardization, in: The Ninth Conference on Computing in Civil and Building Engineering, Taipei, Taiwan, 2002.

[3] A.W. Momber, Hydrodemolition of Concrete Substrates and Reinforced Concrete Structures, Elsevier Applied Science, London, 2005.

[4] The Widest Range of Hydrodemolition Equipment[Online], http://www.Conjet.com (accessed Jan. 1, 2014).

[5] I. Carin, Hydrodemolition of concrete and high-pressure rinsing, Journal Graditelj, Masmedia, Zagreb, 1999.

[6] Ž. Pauše, Probability, Školskaknjiga, Zagreb, 1988.

[7] Vukadinović, The Elements of Probability Theory and Mathematical Statistics, Privrednipregled, Beograd, 1988.

[8] R. Lončarić, The Organization of the Performance of Construction Projects, Engineers and Technucian Association, Zagreb, 1995.

[9] S. Rex, The Technology of Construction, Zagreb, 1990.

[10] Dynamic Plan of Carpentry Material and Optimization of Dimensioning a Performance Phase, Organisation and 
Management in Construction, Dubrovnik, 1991.

[11] Z. Carin, I. Kegelj, I. Miholić, B. Letonija, Database on Renewal of Bridges and Flyovers, Company Carin, Zagreb, 2008.

[12] Ž. Šipek, The Base of Onstruction Works Norm, Company Carin, Zagreb, 2008.

[13] V. Križaić, Erlang distribution for the water robot, in: International Scientific Conference, People Buildings and Environment, Lednice, Czech Republic, Nov. 7-9, 2012.

[14] IGH: C-Data Base for the Bridge at Ivanja Reka, Project
IGH 21-3536/06, Zagreb, 2006-2008.

[15] V. Križaić, Vectoral organization of construction business system, in: 10th International Conference on Organization, Tehnology and Management in Construction, Šibenik, 2011.

[16] Tom Lyche[Online], University of Oslo, 2012, heim.ifi.uio.no/ tom/matrixnormslides.pdf (accessed Jan. 1, 2014).

[17] Vector Norms, Department of Computer Science, Cornell University, 2009. 